\title{
Changing scenario of superficial fungal infection: Emerging resistance, recurrence and remedy in Bangladesh
}

\author{
Mohammad Kamrul Ahsan*1, Mir Nazrul Islam²
}

\begin{abstract}
:
Superficial fungal infections (dermatophytes) affecting skin, nail and hair are the most common infective dermatosis seen in dermatology outpatient clinics. There is a rising prevalence of dermatophytosis, especially in tropical countries like Bangladesh, with a concomitant increase in the number of difficult to treat cases. The reason for this phenomenon is not yet clear, but may be related to inadequate treatment regimen or discontinuation of medication, difficulties in eliminating predisposing factors and sources of re-infection. The pathogenesis and severity of fungal infection depend on various immunological and nonimmunological factors. The rampant use of antifungal therapy in immunocompromised individuals marked the onset of antifungal drug resistance. Fungal resistance can be microbiological or clinical. Genetic alterations in the fungi cause microbiological resistance. Clinical resistance is due to host or drug related factors. All these factors may cause fungal resistance individually or in tandem. Appropriate drug dosing along with antifungal drug susceptibility testing, especially for the dermatophytes isolated from chronic, recurrent cases or those with atypical presentation should be encouraged. Other ways to avoid resistance is the use of combination antifungal therapy. Combination therapy offers advantages in increased synergistic action with enhanced spectrum activity. Finally, newer insights into molecular mechanisms of drug resistance will help in the development of appropriate antifungal therapy. Till then experience based treatment of dermatophytosis is more effective than the standard guideline.
\end{abstract}

Keywords: Dermatophytosis, Fungal resistance, Microbiological, Drug susceptibility.

\section{Introduction}

Fungal infections, including dermatophytes and Candida are the most commonly encountered infection worldwide ${ }^{1}$. It accounts for nearly $25 \%$ of the global skin mycosis, making dermatophytic infections one of the most common types of infective diseases ${ }^{2}$. They belong to three anamorphic genera named as Trichophyton, Epidermophyton, and Microsporum which can be further divided into anthropophilic, zoophilic and geophilic based on their natural habitat. Thus, anthropophilic dermatophytes are associated with humans and rarely infect other animals; zoophilic dermatophytes usually infect animals, but may infect humans who come in contact. Geophilic dermatophytes are generally found in soil and primarily associated with decomposition of keratinous materials such as hair, nails, feathers and horns ${ }^{3}$. When dermatophytes infect humans, they colonize the keratinized outer-most layer of the skin, and usually do not invade the living tissue. The disease is known as tinea or ringworm.

1. Dr. Mohammad Kamrul Ahsan, Associate professor, Dept. of Dermatology, Ibrahim Medical College and BIRDEM General Hospital.

2. Dr. Mir Nazrul Islam, Professor, Dept. of Dermatology, Ibrahim Medical College and BIRDEM General Hospital.

\section{*Corresponding Author:}

Dr. Mohammad Kamrul Ahsan, MBBS, MD (Derma)

Associate Professor, Dept. of Dermatology

Ibrahim Medical College and BIRDEM General Hospital

Shahbagh, Dhaka - 1000, Bangladesh.

Cell phone: +8801703003898

Email: dr.kamrul1966@gmail.com
These infections include tinea corporis, tinea capitis, tinea pedis, tinea cruris and tinea unguium. However, the disease is the result of the host reaction to the enzymes secreted by the fungus during its invasive process.

The tinea infections are prevalent worldwide but they are common in geographical areas with higher humidity. Overpopulation and poor hygienic living conditions also contribute to dermatophytic infections ${ }^{4}$. Hot and humid climate of Bangladesh makes dermatophytosis a very common fungal infection of skin. In recent years, the number of human infections caused by this group of fungi has increased considerably. It is of particular concern in immunocompromised patients as the incidence of systemic and opportunistic fungal as well as candidal infections has increased. The indiscriminate use of antibiotics has also contributed to the installation of fungal infections ${ }^{1}$. Hence, fungal infection has become important cause of mortality and morbidity. On this basis, it is necessary to have antifungal available for the efficient control of fungal infections. Unfortunately, the reason for this fungal resistance phenomenon is not yet clear.

It is assumed that easy availability of cheap, irrational fixed dose corticosteroid antifungal antibacterial combinations in Bangladesh and in vitro resistance to common antifungal is playing a pivotal role. So far, fungal infection is empirically treated and fungal culture and sensitivity is not routinely recommended in Bangladesh. Again clinically successful treatment does not always correlate with the MIC (minimum inhibitory concentration) value of antifungal in vitro. This review expounds upon various possible factors which could have led to such a menace and an emergence of antifungal drug resistance in superficial mycoses. 


\section{Clinical aspects:}

Today, we are facing an onslaught of chronic and recurrent dermatophytosis in volumes never encountered previously. Last 4-5 years, the frequency of such cases has increased alarmingly. The dermatophytosis cases seen in OPD are tip of the iceberg of epidemic that is present in the community. But there is no standard definition for the term "chronic dermatophytosis", although it is described in lay terms as "patients who have suffered from the disease for more than 6 months to 1 year duration, with or without recurrence, in spite of being treated."

Recurrent dermatophytosis refers to the reoccurrence of the dermatophyte infection within few weeks, after completion of treatment ${ }^{5}$. Chronic and recurrent dermatophytic infections cause significant distress to the patients socially, emotionally, and financially.

Again, we are seeing an increased number of atypical presentations with extensive involvement due to increased use of topical corticosteroid preparations. If this happens repeatedly it would lead to multiple active borders with intermittent clearing. The classic description of tinea being circinate with an active erythematous border and central clearing is no longer valid ${ }^{1}$.

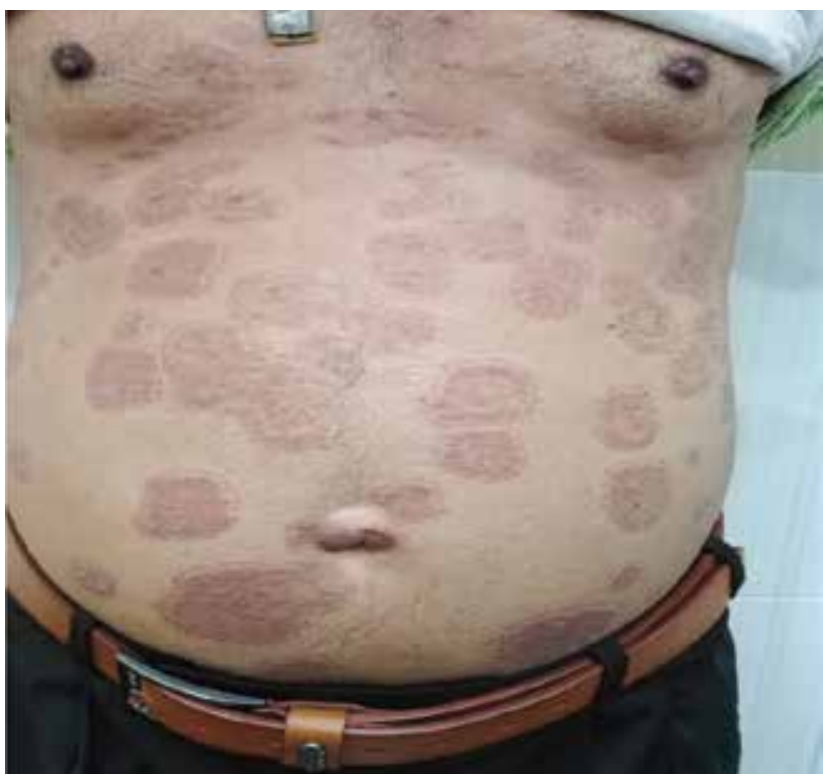

Fig. 1: showing a patient with chronic dermatophytosis. There are multiple circular, circumscribed, slightly erythematous lesions on the trunk.

\section{Patho-mechanisms of chronic dermatophytosis:}

Exact pathogenesis of chronic/recurrent dermatophytic keratin in host. About $90 \%$ cases of chronic dermatophytosis have been attributed to Trichophyton rubrum infection ${ }^{1,5}$. The identity of the pathogen is important for determining a zoonotic reservoir of infection (e.g., cats and dogs for Microsporum canis, cattle for T. verrucosum, and rats for $\mathrm{T}$.

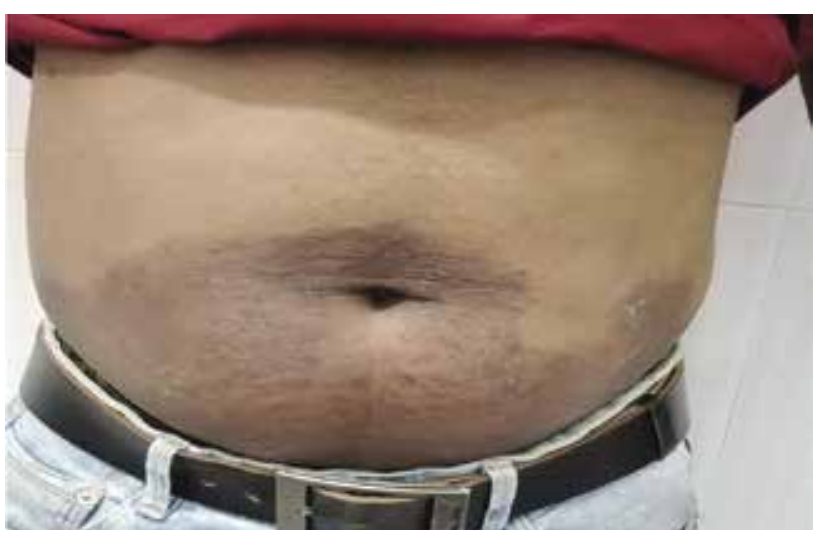

Fig. 2: showing widespread, atypical appearance of tinea involving lower abdomen and groin due to use of topical steroids preparation.

mentagrophytes) ${ }^{1}$. T. rubrum is a resilient fungus with ubiquitous presence. It is capable to evade the host immune system. It survives off the body as a spore until it finds a warm, moist area of skin, where it readily colonizes. Invasion of epidermis by dermatophytes starts with adherence between arthroconidia and keratinocytes, followed by penetration and growth. Arthroconidia adheres to the keratinized tissue and germinates producing hyphae that expand radially in multiple directions $s^{6,7}$. Spores must germinate and penetrate the stratum corneum at a rate faster than epidermal desquamation. This is achieved by several fungal proteases which aid in rapid expansion of dermatophytes. The dermatophytic clearance from the skin is secondary to the activation of cell-mediated immunity and is Th1/Th17 dependant. It is interesting to mention that acute dermatophytosis is associated with Th1 response, where as in chronic dermatophytosis, the immunity is skewed toward Th2 cytokines with high levels of $\operatorname{IgE}$ and IgG4 antibodies ${ }^{7}$. Individuals with defective tilt of immunity toward Th2 cytokines would be more predisposed to chronic dermatophyte infection. This could also explain the increased predisposition to dermatophytosis seen in ichthyosis and atopic population ${ }^{1,8}$. Macrophages and neutrophils migrate in response to fungal invasion of skin and are capable of killing the fungi in normal individuals. Defective phagocytosis of the fungal hyphae is observed in patients with chronic dermatophytosis. Lower free radicals and nitric oxide have been found in patients of chronic dermatophytosis indicating a defective killing mechanism as well. Also CARD9 gene mutation is known to be associated with candida meningitis to recurrent dermatophytic infection of skin and nails 9 .

Patients with immunocompromised status, diabetes mellitus, ichthyosis, atopy and intake of systemic steroids are also predisposed to chronic dermatphytosis. Diabetic patients are more likely to develop onychomycosis after having tinea pedis. Ethnic susceptibility has also been noted, especially in tinea capitis.

Recurrent dermatophytosis is more frequent in low socio-economic group, and tinea corporis and cruris were found to be the most common clinical forms associated with chronicity. Further research is needed to fully understand the 
pathomechanisms of the persistence of dermatophytic infections in certain patients.

\section{Antifungal drug resistance:}

Different studies around the world are noticing an increasing rise in resistance to common antifungal drugs used for the treatment of dermatophytosis ${ }^{10,11}$. Fungal resistance can be defined as microbiologic or clinical resistance or as a composite of the two. Microbiologic resistance refers to nonsusceptibility of a fungus to an antifungal agent by in vitro susceptibility testing, where the MIC of the drug exceeds the susceptibility breakpoint for that organism. Again microbiological resistance may be primary, where resistance is found naturally among certain fungi without prior exposure. Secondary resistance develops among previously susceptible strains after exposure to the antifungal agent and is usually dependent on altered gene expression. Both primary and secondary resistances to antifungal agents have been observed $^{12,13}$.

Clinical resistance has been defined as the persistence or progression of an infection despite appropriate antimicrobial therapy. Such failures can be due to incorrect diagnosis, immunosuppression, and suboptimal dose or duration of therapy.

Antifungal misuse, such as patients often fail to complete the full course of treatment. The inadequate dosage of drugs contributes to the failure in eliminating the disease agent completely, encouraging growth of the most resistant strains. Even low frequency of gene mutation, the selective pressure due to constant use of antifungal agents select a resistant strain that will predominant in the population. Various biochemical mechanisms contribute to the phenotype of drug resistance in fungi ${ }^{14,}{ }^{15}$. Most frequent are decrease in drug uptake, structural alterations in the target site, and an increase in drug efflux in intracellular target levels. Another mechanism of resistance is biofilm production by the dermatophytes. Biofilms are known to confer resistance to both antimicrobial agents and host immunity ${ }^{16}$. So, fungal factors, host factors and drug pharmacology play a role in fungal resistance in isolation or in association with other.

\section{Discussion:}

Dermatologists are perplexed by such complex behavior of dermatophyte infections. Tinea corporis and tinea cruris, once considered to be easy to manage skin conditions, are gradually becoming a dermatologist's nightmare. Clinically, antifungal resistance may be suspected in patients with recurrent episodes of infection, unresponsiveness to first line of therapy, generalized involvement, or atypical form of presentation with usual history of similar lesions in the family. There are no consensus guidelines for the management of these cases; dermatologist are using a combination of oral antifungal, higher doses of antifungal, longer duration of treatment, and even retinoid for the management of these recalcitrant tinea cases. Taking commercial cognizance of fungal infection, here many pharmaceutical companies have started manufacturing terbinafin, itraconazole and even broad spectrum $2^{\text {nd }}$ generation tri-azole antifungal voriconazole.
Unfortunately, we do not have any precise bioavailability studies for most of these formulations. Well-designed studies comparing different antifungal could elucidate the most effective drug as well as dose and duration of therapy ${ }^{17}$. Also, it is need of time to increase our understanding on the molecular mechanisms of antifungal drug resistance and the genetic and host factors. Some predisposing factors relevant to Bangladesh are over-the-counter (OTC) availability and rampant use of topical steroid and antifungal combinations by the patients or unrestricted prescription by quacks and general practitioners. Re-infection from the contacts or fomites may also be a contributing factor, as majority of patients with chronic/recurrent dermatophytosis have multiple affected family members. In case of zoophilic fungal infections, infected animals should be identified and treated. Microsporum canis is the most common form of ringworm in pets $^{1}$.

Overcrowding, sharing of clothes and footwear, poor hygiene, tight clothes, migration of people, obesity, sedentary life style and global warming are also some predisposing factors in Bangladesh. So a successful clinical response to antimicrobial therapy depends not only on the susceptibility of the pathogenic organism, but also relies on the host immune system, drug penetration and distribution, patient compliance, and absence of a protected or persistent focus of infection.

\section{Conclusion:}

Pharmacotherapy is not the only solution for current problems with dermatophytosis. In addition to prudent use of systemic antifungal in proper dosing and duration, topical agents should be applied over the lesions 2 to $3 \mathrm{~cm}$ beyond the border and continued for two week after the rash resolves. Good skin hygiene measures along with simultaneous treatment of other infected family members should be encouraged. Literally, physician's time invested during the first visit for counseling is directly proportionate to the success of the clinical outcome. Antifungal susceptibility test, if possible should be done, it can assist clinician to select the best antifungal regimen. Quality control policy of antifungal drugs should be reviewed as well.

\section{References:}

1. James WD, Elston DM, Treat JR, Neuhaus IM. Diseases resulting from fungi and yeasts. Andrews' diseases of the skin. $13^{\text {th }}$ ed. Elsevier, 2020, pp.291-305.

2. Havlickova B, Czaika VA, Friedrich M. Epidemiological trends in skin mycoses worldwide. Mycoses 2008; 51 suppl 4:2-15.

3. Ellis D, Marriott D, Barton R. Epidemiology: surveillance of fungal infections. Med. Mycol. 2000; 38(sup1):173-82.

4. Niranjan HP, Padmaja N, Priyanka BV. Study of onychomycosis at a tertiary care hospital in South India. J. Evol. Med. Dent. Sci. 2012; 1(5):823-829.

5. Dogra S, Uprety S. The menace of chronic and recurrent dermatophytosis in India: Is the problem deeper than we perceive. Indian Dermatol Online J. 2016; 7(2):73-76. 
6. Martinez-Rossi NM, Peres NT, Rossi A. Pathogenesis of dermatophytosis: Sensing the host tissue. Mycopathologica 2017; 182:215-227.

7. Vermout S, Tabart J, Baldo A, Mathy A, Mignon B. Pathogenesis of dermatophytosis. Mycopathologica 2008; 166: 267-75.

8. Garcia-Romero MT, Arenas R. New insights into genes, immunity, and the occurrence of dermatophytosis. J Invest Dermatol 2015; 135: 655-657.

9. Lanternier F, Pathan S, Vincent QB, Prando C. Deep dermatophytosis and inherited CARD9 deficiency. N Engl J Med. 2013; 369:1704-14.

10. Khatri PK, Kachhawa D, Maurya V, Meena S, Khuller S. Antifungal resistance pattern among dermatophytes in Western Rajasthan. Int. J.Curr.Microbiol. App.Sci 2017; 6(7):499-509.

11. Dogra S, Shaw D, Rudramurthy SM. Antifungal drug susceptibility testing of dermatophytes: Laboratory findings to clinical implications. Indian Dermatol Online J 2019; 10: 225-33.
12. Pai V, Ganavalli A, Kikkeri NN. Antifungal resistance in dermatology. Indian Journal of Dermatology 2018; 63(5): 361-368.

13. Martinez-Rossi NM, Peres NT, Rossi A. Antifungal resistance mechanisms in dermatophytes. Mycopathologica 2008; 166:369-383.

14. Srinivasan A, Lopez-Ribot JL, Ramasubramanian AK. Overcoming antifungal resistance. Drug Discov Today technol 2014; 11: 65-71.

15. Kanafani ZA, Perfect JR. Antimicrobial resistance: resistance to antifungal agents: mechanisms and clinical impact. Clin Infect Dis 2008; 46:120-128

16. Gupta AK, Daigle D, Carviel JL. The role of biofilms onychomycosis. J Am Acad Dermatol.2016; 74:1241-1246.

17. Beardsley J, Halliday CL, Chen SC-A, Sorrell TC. Responding to the emergence of antifungal drug resistance: perspectives from the bench and the bedside. Future Microbiol.2018; 13(10):1175-1191. 